\title{
Téoros
}

Revue de recherche en tourisme

\section{Tourisme et environnement : des actions internationales}

\section{André Hut}

Volume 10, numéro 1, mars 1991

Tourisme et environnement

URI : https://id.erudit.org/iderudit/1079820ar

DOI : https://doi.org/10.7202/1079820ar

Aller au sommaire du numéro

Éditeur(s)

Université du Québec à Montréal

ISSN

0712-8657 (imprimé)

1923-2705 (numérique)

Découvrir la revue

Citer cet article

Hut, A. (1991). Tourisme et environnement : des actions internationales.

Téoros, 10(1), 43-45. https://doi.org/10.7202/1079820ar d'utilisation que vous pouvez consulter en ligne.

https://apropos.erudit.org/fr/usagers/politique-dutilisation/ 


\section{EUR-INFO \\ Tourisme et environnement: \\ André Hut* des actions internationales}

\section{UNESCO: un fondement éthique!}

La Convention (1972), l'action et la sensibilisation de l'UNESCO en faveur de la protection du patrimoine mondial, culturel et naturel, impliquent la majorité des pays du continent européen qu'ils aient ratifié la convention ou bénéficient de sites etmonuments inscrits sur la liste du patrimoine mondial. Parmi eux, des pays européens ont présenté un site industriel, la France avec les Salines Royales d'Arcet Senans, la Pologne avec le Musée des techniques et du travail dans les mines de sel de Wieliczkaet le Royaume-Uni avec la Gorge d'Ironbridge.

En outre, le Comitế du Tourisme d'ICOMOS - Conseil international des monuments et des sites - $s^{\prime}$ est senti concerné par les effets positifs et négatifs qu'entraîne pour ce patrimoine le développement des activites touristiques dans le monde. A Bruxelles, en novembre 1976, il a rassemblé les grandes organisations mondiales, gouvernementales ou non, se préoccupant du tourisme pour des raisons professionnelles et économiques ainsi que culturelles, éducatives et sociales afin de s'engager en commun à signer, proclamer et défendre la Charte du tourisme culturel.

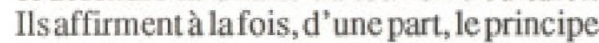
que "c'est le respect du patrimoine mondial, culturel et naturel, qui doit prévaloir sur toute autre considération si justifiée qu'elle puisse être du point de vue social, politique ou économique" et, d'autre part, l'action qui en découle "d'adopter toutes les mesures appropriées de sensibilisation,

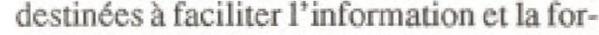
mation des personnes se déplaçant à des fins touristiques" tant du côté des usagers que des professionnels, cadres et animateurs. (I)

Enfin, l'UNESCO a lancé la décennie mondiale du développement culturel (19881997). "L'objectif essentiel est de rendre aux valeurs culturelles et humaines une place centrale dans le développement technique etéconomique", ainsi que le synthétise l'éditorial d'un courrier de l'UNESCO consacré à ce sujet ${ }^{(2)}$. Comme l'affirme le directeur général Mayor Zaragoza: "Un

Expert en tourisme, professeur et animateur, André Hut est correspondant de Táoros en Europe véritable développement doit donc, en dernière analyse, puiser ses priorités, ses motivations et ses finalités dans la culture. Mais jusqu'ici, ce constat s'est surtout fait par défaut. Cette exigence ne s'impose pas uniquement dans les pays du Tiers-Monde [mais] aussi, de plus en plus, dans les pays industriels, où la course effrénée à la croissance des biens matériels se poursuit au détriment des dimensions spirituelle, éthique et esthétique de la vie,comme aussi au prix de graves déséquilibres entre l'homme etl'environnement... Une liaison maîtrisée entre la culture et le développement peut permettre, à la fois, d'approfondir les identités créatrices, de bloquer les phénomènes d'uniformisation, de favoriser une égalisation croissante des chances d'expression des différentes cultures et, par là, de promouvoir des échanges de plus en plus enrichissants entre elles... une diversité qui unit, une créativité qui rapproche, une solidarité qui libère...".

Cette dimension culturelle du développement-notamment du tourisme au seinde celui-ci comme aussi de celle-ci au sein du développement touristique - était déjà formellement proclamée dans la Déclaration de Manille, en 1980, de l'Organisation mondiale du tourisme. "Dans la pratique du tourisme, leséléments spirituels doivent l'emporter sur les éléments techniques et matériels. Ces éléments spirituels sont fondamentalement les suivants: le plein épanouissement de la personne humaine, une contribution sans cesse accrue à l'éducation, l'égalité du destin des peuples, la libération de l'homme dans le respect de son identité et de sa dignité, l'affirmation de l'originalité des cultures et le respect du patrimoine moral des peuples [21]... Toutefois, la rentabilité économique du tourisme ne saurait constituer le seul critère d'appréciation de l'encouragement que les États doivent apporter à cette activité. Le droit aux vacances, l'ouverture du citoyen à la connaissance de son propre milieu, l'approfondissement de saconscience nationale et de la solidarité qui le lie à ses compatriotes, le sens de son appartenance à une culture et à un peuple sont autant de raisons impérieuses de lui favoriser par l'accès aux vacances et aux voyages sa participation au tourisme national et international [8]."
Cela explique l'adoption par la Conférence générale de l'UNESCO en 1987, d'un projet symbolique mettant en oeuvre activement l'esprit de la décennie du développement culturel; L'étude intégrale des routes de la SOIE: les routes du dialogue. Ils'agira d'une approche pluridisciplinaire des trois routes principales, terrestres et maritimes, qui ont suivi durant 2000 ans des "caravanes qui ne transportaient pas que de la soie mais aussi quantité de produits naturels et d'articles manufacturés, du bronze à la porcelaine en passant par la laine et le cobalt. Ces routes qui se prolongeaient par voie de terre ou de mer étaient des voies par lesquelles passaient les idées, les techniques, les religions et les arts, permettant ainsi aux civilisations de se féconder mutuellement, les échanges ne se faisant pas à sens unique (le long) de ces cheminscaravaniers qui reliaientl'Orient à I'Occident, ces grandes artères d'échanges commerciaux et culturels". En 1992, les cinq années de recherches et d'actions se clôtureront à Paris avec une Conférence internationale qui fera le point sur les différentes études et une grande exposition présentant d'importantes collections réunies pour la première fois ainsi que la publication d'un Atlas historique des Routes de la Soie.

Le Conseil de l'Europe prend le relais sur le continent et la plupart des pays européens font l'étude de la soie sur leur territoire et son rôle dans l'économie, la culture, le commerce; de plus, ils dressentl' inventaire des musćes et centres touristiques liés à la soie et à l'industrie séricicole, proposant chacun un itinéraire culturel de la soie ${ }^{[3]}$.

\section{Conseil de l'Europe: une synergie bénéfique!}

Le Conseil de l'Europe, fondé en 1949, et regroupant 23 pays du continent, mène aussidepuis 1963 une action en faveurde la sauvegarde et de la mise en valeur du patrimoine, contribuant ainsi à l'amélioration du cadre et de la qualité de la vie.

En 1969, à Bruxelles, sous la présidence du Prince de Liège, le Conseil de la coopération culturelle a réuni, en novembre, les ministres responsables de la protection du 
patrimoine culturel immobilier afin d'élaborer une politique active dans ce domaine. Parmi les raisons qui justifient cette politique, le ministre espagnol a cité, outre l'expansion démographique et

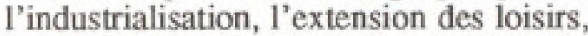
le tourisme de l'avenir qui prendra un aspect culturel dominant et la valeur éducative de ce patrimoine ${ }^{(4)}$.

Afin de sensibiliser l'opinion publique, le Conseil de 1'Europe lançait, en 1975, une Campagne européenne du patrimoine architectural, qui eut un grand retentissement quant à "la conservation du patrimoine architectural, industriel, il n'a pu se concevoir que dans le contexte d'un élargissement progressif du concept même de patrimoine. De proche en proche, l'architecture rurale, les constructions techniques et l'archéologie industrielle furent-ellesprises en considération au même titre qu'une cathédrale ou un château, à l'intérieur d'une nouvelle conception de la conservation dite INTÉGRÉE."(?)

D'ailleurs, la Convention pour la sauvegarde du patrimoine architectural de l'Europe, adoptée en 1985 par les ministres responsables de ce domaine rassemblés en conférence à Grewarde, a souligné ce changement tant quantitatif que qualitatif du patrimoine, Dès 1979 d'ailleurs, l'assemblée parlementaire du Conseil de l'Europeavaitadopté une "recommandation relative à l'archéologie industrielle".

En conséquence, le Conseil de 1'Europe allait organiser une série de colloques sur le sujet: à Lyon/Vaulx-en-Velin en 1985 , Quelle politique pour le patrimoine industriel; en 1986, à Madrid, Les ouvrages publics: une nouvelle dimension du patrimoine; en 1988,à Bochum, Les monuments techniques de la mine, patrimoine culturel, et en 1989, à Londres, Les outils de la mémoire.

En effet, d'une part, "il s'agissait d'envisager les ouvrages d'art et de génie civil en tant que nouvelle dimension du patrimoine. Les ponts, fortifications, aqueducs, canaux, barrages, phares, installations portuaires, gares de chemin de fer, etc., apparaissent en effet, au même titre que tel ou tel monument célèbre, comme les signes d'une identité culturelle européenne. Ils ont fortement marqué le paysage, contribuant à lui donner sa physionomie actuelle. Ils ont souvent joué un rốleprépondérant dans l'histoire. Routes et ponts voies commerciales ou militaires, routes de pèlerinages - ont tissé un réseau de relations denses entre tous les pays européens. "1(6)
D'autre part - mais on pourrait en dire autant du textile, du ciment, de la métallurgie ou de la verrerie..." les monuments techniques de la mine. Avec le charbon et le fer - mais aussi l'étain, le zinc, le plomb, lecuivre... - on touche aux fondements de la révolution industrielle. Des activités qui ont marqué toute l'Europe, déterminé toute la vie de certaines régions ${ }^{(n)}$,

Les révolutions technologiques et énergétiques des quinze demières années ainsi que les stratégies industrielles européennes et internationales ont amené des fermetures de mines, des faillites ou des déplacements d'entreprises industrielles vers d'autres sites, extra-urbains - de zonings industriels ou de littoraux. D'où bấtiments, sites et friches industrielles à l'abandon. Quelle politique? Démolition, réaffectation, choix parmi des entreprises similaires, valorisation culturelle et touristique?

Cetie problématique vis-à-vis du patrimoine industriel n'aurait pas abouti au Conseil de l'Europe et à des prises de décisions par les ministres responsables rassemblés en conférences s'il n'y avait pas eu une multiplication d'initiatives diverses et convergentes de sauvetage et de mise en valeur de celui-ci. Le musée d'Ironbridge Gorge, en Grande-Bretagne, inauguré en 1968 en est une des plus remarquables. C'est là que s'est tenu, en 1973, le premier Congrès international d'archéologie industrielle surtout orienté vers les monuments industriels. Il fut suivi de celui de Bochum, en Allemagne, préoccupé notamment de la sauvegarde des aspects sociaux du patrimoine industriel tandis que le troisieme en Suède a abouti à la fondation du Comité international pour la conservation du patrimoine industriel (TICCIH). Celui-ci allait bientôt alimenter et être renforcé par l'action du Conseil de l'Europe dans ce domaine.

De musées d'une activité industrielle sauvant et valorisant le site même de la production en écomusées de sites industriels comme celui du Creusot, on en arriva à parler de towrisme industriel et un Colloque national sur les enjeux de celui-ci se tint à Besançon, en novembre 1985, organisé par l'Association comtoise des arts et traditions populaires: ses enjeux scientifiques, culturels, économiques et sociaux sans omettre l'expérience des agences de voyages en ce domaine. Rappelons les initiatives des Itinéraires culturels de la soie, du Conseil del'Europe et de sespays membres (Loreto, 21).

Le 6e Congrès international du TICCIH, à Vienne en septembre 1987, allait aborder notamment les efforts entre les monuments industriels et le tourisme. "Les débats furent marqués par les préoccupations de notre temps à savoir la conjoncture économique difficile et le désir, pour les promoteurs et les animateursd'archéologie industrielle, de rentabiliser au maximum leurs initiatives et les sites dont ils assurent la mise en valeur. Ce souci en entraîne un autre: celui de garder l'équilibre entre la conservation des vestiges industriels dignes d'intérêts et les nécessaires aménagements en vue de l'accueil des touristes... Le poids de l'opinion publique en vue d'influencer les autorités dans le sens d'une politique de conservation est essentielle dans le contexte d'une civilisation de loisirs. Il fut aussi question de la formation de guides spécialisés en archéologie industrielle.

De son côté, la mềme année, l'Association internationale des experts scientifiques du tourisme (AIEST), abordait lors de son 34e Congrès, à Prague en septembre 1984, Tourisme et patrimoine bâti: aspects culurels, juridiques, économiques et promotionnels ${ }^{(\omega)}$. II faut mettre en évidence les remarquables analyses du groupe des experts Français J.-C. Croizi et V. Patin sur la "signification culturelle et la valorisation touristique du patrimoine bâti", notamment pour le tourisme rural (S. Thibal) ou le tourisme social (A. Guignand). Monsieur Planque a explicité d'une manière concrète les pratiques et les politiques du Fonds d'intervention culturelle par lequel l'État distribue son aide à des initiatives locales et privées ainsi que les initiatives de la Caisse nationale des monuments historiques. établissement public sous tutelle du ministêre de la Culture, chargé de l'accueil et de l'information du public etplus généralement de l'animation du patrimoine monumental français; ces politiques mettent en oeuvre les décisions du ministre de la Culture, J. Lang, dont il sera mention plus loin.

Parallèlement, la Conférence permanente des pouvoirs locaux et régionaux de l'Europe s'est préoccupée dès le début des "villes d'intérết historique", notamment à l'occasion de la Confrontation européenne des villes d'intérét historique à Split (Yougoslavie), en 1971. Celle-ci a été prépparée par une série de rencontres abordant successivement les problèmes d'inventaire, de conservation, de réanimation, de financementet de mise en valeur du patrimoine immobilier historique des villes ${ }^{(11)}$.

Le 6e Symposium européen des villes historiques, qui s'est déroulé à Cambridge (UK) en septembre 1989, a traité exclusivement des problèmes particuliers au Tourisme dans les villes historiques, avec ses effets bénéfiques et négatifs appelant 
une gestion des flux du trafic et des personnes ainsi qu'une politique foncière, économique et touristique en faveur tant des visiteurs que des populations d'accueil ${ }^{(12)}$. La Fédération internationale des associations pour la sauvegarde du patrimoine naturel et culturel de l'Europe, EUROPA NOSTRA, fondée en 1963, s'était déjà préoccupée de tourisme et conservation, notamment lors de sa Conférence annuelle à Malte en 1970.

Patrimoine architectural et tourisme, tel fut le theme du Colloque qu'ICOMOSWALLONIE a tenu à Spa, station thermale historique, en octobre $1986 .{ }^{~ " L} \mathrm{~L}$ 'heure est manifestementà un rapprochement des deux secteurs. Il estgrand temps qu'ils comprennent leur complémentarité tant sur le plan économique que culturel!'?(1)

En effet, trop souvent jusqu'ici le patrimoine et le tourisme relevent des compétences de ministres etd'administrations différentes. Même au sein du ministère de la Culture, il $n^{5} y$ a pas nécessairement des vases communicants entre le département responsable du patrimoine et celui de l'education populaire et de l'animation. A fortiori - et c'est notre expérience de 30 ans - pour l'animation touristique dont la culture rejette la responsabilité sur le tourisme et vice-versa ou prend une initiative sans la collaboration de l'autre. Enfin, les choses commencent à changer. La France a joué à nouveau un rồle de précurseur car, en 1989, le ministre Jack Lang a signé une Convention culture-tourisme avec le ministre du Tourisme, Olivier Stim. "Ce texte prévoit un certain nombre de mesures pour intensifier et diversifier les flux de touristes. Elles portent notamment sur la signalisation touristique routière, l'accueil dans les monumentshistoriques, 1 "utilisation nouvelle du patrimoine, la mise en valeur des grands monuments de l'Hexagone et d'itinérairesculturels thématiques, ainsi que le développement d'actions significatives dans le domaine de laculture scientifiqueet technique." ${ }^{\text {(r14) }}$ En Belgique, alors que le tourisme dépendait, depuis les réformes institutionnelles de fédéralisation, des communautés culturelles et linguistiques (français, flamand, allemand), la présence du patrimoine, du tourisme, des secteurs d'education populaire et d'animation chez le même ministre et le même ministère... n'ont pas amélioré les synergieset les actions concertés $^{(15)}, \boldsymbol{f}$

\section{Notes oxplicatives}

(1) LaConventiondu patrimoine mondial, Le journaliate du tourisme, FIJET, no 38, 3/87.

(2) Le Courriar da I'UNESCO, nowembre 1989

(3) Idem, "A la redecouverte des routes de la soie"; "Routes de la soie, chemins de la connaissance". in Courrier de I'UNESCO. 3/1989. Loreto, Patrimoine ot itinaraires culturals européens, Bruxelles, 1990.

(4) La libre Belgique, 27/11/1969

(5) KERREMANS, R. "Le patrimoine industriel et le Conseildel'Europe", in L'archéologie industrielle. nouvelles du patrimoine, nos $23 / 24$, decembre 1988 , pp. 14-15.

(6) Ilbid.

(7) lbid

(16) Les enjeux du tourisme industriel, Aetes du Colloque national, 14-15/11/1985, Besançon; Archobologia ot industrio, dossiers histoire t musóologie, Dijon, no 107, 7/8/1966; Images de l'Ecomusée, Museum, no 148, 1985, UNESCO. Paris: Loreto, Patrimolne et itineralires culturals auropáons, no 21, 1990. Bruxelles, 2éme partie, chapitre V. Archéologie industrielle. en Belgicue trancophone: I'introduction. "Aperçu historiographique general". J.P. Hendrickx in Wallonie-Bruxelles: berceau de I'industrie sur le continent europeen, Lourvain-La-Neuve, 1990.

(9) GAIER, C. Patrimoine industriel et tourisme, Lisge, nos 9/10, 1987. TICCICH Secretary: SMITH, Stuart B., Ironbridge Gorge Museum Trust. Ironbridge, Telfford, Stropshire, TFG-7AW, Great Britain.

(10) Tourisme et patrimoine bâti - aspects culturels. furidiques, economiqueset promotionnels, Rapport du 34 e Congràs de I'AIEST, 9-15/9/1984, Éditions AlEST, St Gall, Suisse, wol . 25; Revue dutourisme, no 4, oct./dec. 1984, Varnbūchtrasse, 19, Ch-9000, St Gall.

111) Confrontationeuropénenedes villes historiques, Split, Yougoslavie, 20-23/10/1971, CPLIP(B) 35 \& 36 - Conseil de l'Europe, Strasbourg.

(12) 6e Symposium europeen des villes historiques: Villes historiques et tounisme. Cambridge. UK, 20 22/9/1989; Conseil de l'Europe, Conf/Hist (B9). Strasbourg.

(13) Colloque patrimoine architectural et tourisme, Spa, oct. 1986. Les Actes à la Fondation Rei Baud ouln, 21 ruede Brederode, B1000, Bruxelles; Patrimoine et tourisme - nouvelles du patrimoine, no 12,12 i 1986-2/1987; adresse: 17 , avenue General de Gaulle, 7050 Bruxelles.

(14) Le Monde, 11 mars 1989, Une convention culture: tourisme.

(15) Cf, pour le Quebec et la Communaute francaise de Belgique, Teoros, supplement, automne 1987. A Hut, pp. 8-9 et 16-18. 